\title{
Autonomie als Prädiktor intrinsischer Motivation im Schulsport
}

\section{Eine Person $\times$ Situation-Perspektive}

\author{
Vanda Sieber ${ }^{1}$, Mirko Wegner ${ }^{2}$ und Julia Schüler ${ }^{3}$ \\ 1 Psychologisches Institut, Universität Zürich \\ ${ }^{2}$ Institut für Sportwissenschaft, Universität Bern \\ ${ }^{3}$ Institut für Sportwissenschaft, Universität Konstanz
}

\begin{abstract}
Zusammenfassung: Autonomieförderung gilt als ein wichtiger Prädiktor für intrinsische Motivation im Sport. Anlehnend an bestehende Arbeiten, die zeigen, dass der Zusammenhang von Autonomie und Motivation durch Unterschiede in der dispositionellen Autonomieneigung moderiert wird, prüft die vorliegende Arbeit die zugrundeliegende Passungshypothese für intrinsische Motivation direkt im Schulsportunterricht. Es wird angenommen, dass autonomieförderliche Instruktionen besonders für Schüler und Schülerinnen mit starker Autonomiedisposition die intrinsische Motivation für den Schulsport begünstigen, während sie für Personen mit schwächerer Autonomieausprägung weniger wirksam sind. In einem experimentellen Design wurden 45 Schülerinnen und Schülern entweder einer Autonomiefördernden (AF) Bedingung, einer Autonomie-einschränkenden (AE) Bedingung oder einer Kontrollgruppe (KG) zugeordnet und die intrinsische Motivation bei einer Basketballübung erfasst. Wie erwartet berichten Personen mit stark ausgeprägter Autonomiedisposition eine signifikant höhere intrinsische Motivation in der Autonomieförderungs bedingung als Personen mit schwacher Autonomieausprägung. Die Ergebnisse sprechen für eine differentielle Betrachtung von Autonomieförderung im Schulsport.
\end{abstract}

Schlüsselwörter: Autonomieunterstützung, Autonomiedisposition, intrinsische Motivation, Schulsport

Autonomy as a Predictor of Intrinsic Motivation in Physical Education Classes: A Person $\times$ Situation Perspective

Abstract: Autonomy support is considered as an important predictor of intrinsic motivation in sport environments. Referring to previous studies, which showed that the relationship between autonomy and motivation is influenced by an autonomy disposition, we aim to test the underlying fit hypothesis of intrinsic motivation in physical education classes. It is assumed that autonomy-supportive instructions are especially beneficial for people with a strong autonomy disposition, in contrast to people with a weaker autonomy disposition. In an experimental design, 45 participants were assigned to either an autonomy-supportive (AF) condition, an autonomy-restrictive (AE) condition, or a control condition. After the experimental manipulation, intrinsic motivation in a basketball-based task was assessed. As assumed, people with a strong autonomy disposition showed a significantly higher intrinsic motivation in the AF group compared with participants with a weak autonomy disposition. Taken together, our results support a differential view on autonomy support in physical education classes.

Keywords: autonomy support, autonomy disposition, intrinsic motivation, physical education

Die wachsende sportliche Inaktivität stellt ein großes gesellschaftliches Problem dar (European Commission, 2014; Lamprecht, Fischer \& Stamm, 2014) und verlangt nach einer Analyse der Prädiktoren für körperliche Aktivität. Ein wichtiger Prädiktor für sportliche Aktivität im Erwachsenenalter ist die Aktivität im Kindes- und Jugendalter (Fuchs, 2003), wobei Studien, die die körperliche Aktivität über die Lebensspanne hinweg betrachten, genau in diesem Alter eine Abnahme der körperlichen Aktivität aufzeigen (Caspersen, Pereira \& Curran, 2000; van Mechelen, Twisk, Post, Snel \& Kemper, 2000; Telama \& Yang, 2000).
Um Kinder und Jugendliche im Sportunterricht zu motivieren, wurde bislang überwiegend auf situative Faktoren, wie beispielsweise autonomieförderliche Sportumwelten fokussiert (Adie, Duda \& Ntoumanis, 2008; Fenton, Duda \& Barrett, 2016). In der vorliegenden Arbeit setzten wir an den Lewin'schen Grundgedanken an, dass sich die Motivation von Personen am besten aus der Betrachtung des Zusammenspiels von Merkmalen in der Situation und Merkmalen in der Person erklären lässt (Person $\times$ Situation Ansatz, Lewin 1931, 1936). Wir formulieren eine Passungshypothese (s. auch Schüler, Sheldon, Prentice \& Halusic, 2014), nach der autonomieför- 
derliche Sportumwelten vor allem dann die Motivation der Sporttreibenden erhöht, wenn auf Seiten der Person eine korrespondierende hoch ausgeprägte Autonomiedisposition vorliegt.

\section{Selbstbestimmungstheorie und Autonomieunterstützung}

Der Fokus der Selbstbestimmungstheorie (Deci \& Ryan, 1985, 2000) liegt darauf, Umwelten zu identifizieren, die die Befriedigung von Basisbedürfnissen (Autonomie, Kompetenz, soziale Eingebundenheit) ermöglichen. So können beispielsweise Personen in hierarchisch höheren Positionen (z.B. Sportlehrpersonen, Trainer) durch ihr Verhalten autonomiefördernde Lernumwelten, z.B. durch das Anbieten von Wahlmöglichkeiten, erzeugen (Hagger \& Chatzisarantis, 2015; Ryan \& Deci, 2008). Ein häufig verwendeter Fragebogen zur Erfassung der Autonomieunterstützung ist der Health Climate Questionnaire (Williams, Niemiec, Patrick, Ryan \& Deci, 2009). Er umfasst Verhaltensbereiche, wie das Beantworten von Fragen und das Anbieten von Wahlmöglichkeiten. Die positiven Effekte von Autonomieunterstützung zeigen sich im Arbeits- (Baard, Deci \& Ryan, 2004; Güntert, 2015; Lynch, Plant \& Ryan, 2005; Nie, Chua, Yeung, Ryan \& Chan, 2015; Williams et al., 2009) und Schulkontext (Hagger \& Chatzisarantis, 2015; Sheldon \& Krieger, 2007; Vansteenkiste, Simons, Lens, Soenens \& Matos, 2005). Auch Studien im Sportkontext zeigen, dass autonomieunterstützendes Verhalten von Sportlehrpersonen mit mehr Motivation, höherem Wohlbefinden und einer größeren Persistenz beim Training einhergeht (Adie et al., 2008; Adie, Duda \& Ntoumanis, 2012; Edmunds, Ntoumanis \& Duda, 2006; Fenton et al., 2016; How, Whipp, Dimmock \& Jackson, 2013). Da in der Selbstbestimmungstheorie das Erleben von Autonomie als ein universales Grundbedürfnis konzeptualisiert ist, wird individuellen Unterschieden kaum Beachtung geschenkt (Schüler, Brandstätter \& Sheldon, 2013).

Ganz im Gegensatz dazu, spricht die Motiv-Dispositionstheorie (MDT, McClelland, 1985) individuellen Unterschieden in Motiven eine starke motivations- und verhaltenserklärende Wirkung zu. Sie postuliert u.a., dass situative Rahmenbedingungen nicht für alle gleichermaßen stark wirken, sondern unterschiedlich starke Anreiz- und Befriedigungswirkung in Abhängigkeit von der Motivausprägung haben. Hieraus lässt sich eine Passungshypothese ableiten, die im Folgenden erläutert wird.

\section{Die Passungshypothese - Individuelle Unterschiede im Autonomiemotiv}

In ihren Arbeiten zur Passungshypothese weisen Schüler und Kollegen (Schüler \& Brandstätter, 2013; Schüler et al., 2013; Schüler, Sheldon \& Fröhlich, 2010; Schüler et al., 2014; Sheldon \& Schüler, 2011; Sieber, Schüler \& Wegner, 2016) unter anderem darauf hin, dass individuelle Unterschiede in der Ausprägung impliziter Motive (McClelland, 1985) beeinflussen, wie stark Personen von Autonomieunterstützung in der Sportumwelt profitieren. Das Autonomiemotiv bezeichnet dabei eine stabile Präferenz, Freiheit zu erfahren und das chronische Bestreben sich selbst in diversen Situationen als handelnde Akteurin zu erleben (DeCharms, 1968; Schüler et al., 2014). Wie für andere implizite Motive, sind auch für das implizite Autonomiemotiv der Kern unbewusste und affektbasierte Präferenzen für motivrelevante Anreize (z. B. sich unabhängig fühlen) (Schüler et al., 2014). In zwei Studien konnten Schüler und Kollegen (2014) zeigen, dass Autonomieunterstützung insbesondere dann vorteilhaft für Motivation und Wohlbefinden ist, wenn Personen ein moderates oder hohes implizites Autonomiemotiv besitzen, aber nicht, wenn sie ein niedriges Autonomiemotiv aufweisen. Dieses Resultat bestätigt die oben genannte Passungshypothese (z.B. Hofer \& Busch, 2011; Schüler et al., 2010, 2013; Sheldon \& Schüler, 2011), die besagt, dass Personen dann profitieren, wenn ihre Persönlichkeit (z. B. Autonomiemotiv) mit der sozialen Umgebung übereinstimmt (z. B. Möglichkeit, selbstständig zu handeln). Die Unterschiede kommen gemäß den Autoren dadurch zustande, dass Personen aufgrund ihrer impliziten Motive Autonomie als unterschiedlich belohnend empfinden.

Die vorliegende Studie überträgt die Passungsannahme auf den Schulsportkontext und prüft sie experimentell. Aufgrund der Wichtigkeit intrinsischer Motivation für aktuelles und zukünftiges Sportverhalten wird diese als abhängiges Maß erfasst.

\section{Methode}

\section{Versuchspersonen}

Fünfundvierzig Jugendliche nahmen an dem Experiment teil, das innerhalb einer Sportlektion (90 Minuten) an der Schule durchgeführt wurde. Die Teilnehmenden und deren Eltern haben vor der Studie eine Einverständniserklärung 
unterschrieben. Von den 45 Jugendlichen waren 16 weiblich, das Durchschnittsalter betrug 14.2 Jahre $(S D=1.0)$.

\section{Studiendurchführung}

Die Jugendlichen erhielten zu Beginn der Untersuchung einen Fragebogen zur Messung des Autonomiemotivs und einer Baseline-Messung ihrer intrinsischen Motivation. Daraufhin wurden die Jugendlichen zufällig den Versuchsgruppen Autonomieförderung (AF, $n=17$ ) und $\mathrm{Au}$ tonomieeinschränkung (AE, $n=14)$ sowie der Kontrollbedingung (KG, $n=14$ ) zugeteilt. Der Sportlehrer organisierte im Rahmen der Unterrichtsstunde ein Basketballspiel. Im Verlauf der Stunde wurden die Untersuchungsteilnehmenden einzeln aus dem Spiel gerufen und warteten fünf Minuten auf einer Bank am Spielfeldrand. Die Schülerinnen und Schüler wurden dann instruiert mittels unterschiedlicher Basketballwürfe (Sprungwurf, Freiwurf, Korbleger) innerhalb von drei Minuten eine möglichst hohe Punktzahl zu erzielen.

Die unterschiedlichen Untersuchungsbedingungen wurden in Anlehnung an die Iteminhalte eines Fragebogens zur Erfassung von Autonomieförderung (Health Climate Questionnaire; Williams, Grow, Freedman, Ryan \& Deci, 1996) gestaltet. Die Untersuchungsbedingungen variierten einerseits hinsichtlich der Autonomiegewährung durch die Instruktionen, welche die Schülerinnen und Schüler erhielten. Zusätzlich wurde Autonomie durch die Rahmenbedingungen, in denen die Übung durchgeführt wurde, variiert. Im Folgenden wird detailliert auf die Gestaltung der experimentellen Bedingungen eingegangen.

Autonomieförderung (AF). Für die Autonomieförderungsgruppe waren drei nummerierte Hütchen auf dem Basketballfeld positioniert, von denen jeweils die Würfe ausgeführt wurden, die vor dem Beginn der Übung kurz von der Versuchsleitung vorgezeigt wurden. Um einen möglichst hohen Grad an Autonomie zu gewähren, wurde mithilfe von Formulierungen wie „du kannst“ oder „du möchtest" gearbeitet. Die Instruktionen waren angepasste Items des Health Climate Questionnaire (Williams et al., 1996). Die Probanden wurden zum Beispiel ermutigt Fragen zu stellen und es wurde ihnen angeboten zu wählen, von wo aus sie welche Würfe ausführen wollten (z. B. „Du kannst wählen, von wo aus du welchen Wurf ausführen möchtest.").

Autonomieeinschränkung (AE). Auch für die autonomieeingeschränkte Gruppe waren drei nummerierte Hütchen im Basketballfeld aufgestellt. Hier waren die Hütchen jedoch nicht nur maßgebend für die Distanz von der aus der Wurf ausgeführt werden musste, sondern bei jedem Hütchen musste ein spezifischer Wurf ausgeführt werden (Position 1: aus dem Sprung; Position 2: aus dem Stand;
Position 3: Korbleger- für Linkshänder wurden Position 1 und 3 vertauscht). Während der Übung griff die Studienleitung immer wieder ein, indem sie dem Schüler oder der Schülerin sagte, was sie genau zu tun hat. Zusätzlich war bei dieser Gruppe das Feld um die Hütchen durch Markierungen am Boden eingegrenzt (1 x $2 \mathrm{~m})$, in denen sich der Schüler beim Wurf befinden musste. Die Größe des Feldes war so gewählt, dass sie keine Einschränkung auf die Durchführung der Übung hatte. Die Autonomieeinschränkung wurde durch die Wortwahl in der Instruktion verschärft (z. B. „Du musst jetzt von hier werfen!“).

Kontrollgruppe (KG). Die Übung war auch für die Kontrollgruppe dieselbe. Wobei wie bei der autonomieeinschränkenden Bedingung die Position und Wurfart durch die Versuchsleitung vorgegeben wurden. Ein wichtiges Unterscheidungsmerkmal zur autonomieeinschränkenden Gruppe war jedoch, dass die Versuchsleitung eine neutrale Sprache angewendet hat, um die Übung zu beschreiben (z.B. „Die kommende Übung hat zum Ziel während drei Minuten möglichst viele Körbe zu erzielen. Mit drei Hütchen sind die Positionen markiert, von wo aus der Ball geworfen wird“). Es wurden Antworten auf Fragen gegeben, jedoch wurde nicht explizit dazu aufgefordert Fragen zu stellen.

Nach der experimentellen Induktion von Autonomieförderung vs. Autonomieeinschränkung, respektive der neutralen Kontrollbedingung, erfolgte die Messung der abhängigen Variable intrinsische Motivation in Abhängigkeit der zuvor durchgeführten Übung. Danach gingen die Schülerinnen und Schüler wieder in den regulären Sportunterricht zurück.

\section{Messinstrumente}

Implizites Autonomiemotiv. Das implizite Autonomiemotiv wurde mit der häufig zur Erfassung impliziter Motive verwendeten Bildgeschichtenübung (PSE; Schultheiss \& Pang, 2007) erfasst. Die Teilnehmenden wurden gebeten Geschichten zu vier Bildvorlagen zu schreiben (Kapitän, Boxer, Paar am Fluss, Mann und Frau in der Bar). Dabei war die Instruktion, das Bild jeweils für 15 Sekunden anzuschauen und dann innerhalb von vier Minuten eine Geschichte mit einem Anfang, einem Mittelteil und einem Ende zu verfassen. Die Geschichten wurden daraufhin mithilfe des Kodiersystems von DeCharms und Plimpton (1992; siehe auch Schüler et al., 2014) ausgewertet. Das Autonomiemotiv wurde immer dann kodiert, wenn in den Texten (a) selbstbestimmte Zielsetzungen, (b) instrumentelle Aktivitäten zur Zielerreichung, (c) Realitätswahrnehmungen, (d) persönliche Verantwortungen, (e) Selbstvertrauen oder (f) persönliche Verursachung erwähnt wurden. Die Geschichten wurden von zwei erfahrenen Ko- 
Tabelle 1. Hierarchische Regression mit intrinsischer Motivation zum Zeitpunkt T2

\begin{tabular}{|c|c|c|c|c|c|c|c|c|c|}
\hline \multirow[b]{2}{*}{ Variable } & \multicolumn{3}{|c|}{ Modell 1} & \multicolumn{3}{|c|}{ Modell 2} & \multicolumn{3}{|c|}{ Modell 3} \\
\hline & $B$ & SE B & $p$ & $B$ & SE B & $p$ & $B$ & SE B & $p$ \\
\hline Baseline intrinsische Motivation & .578 & .197 & .005 & .720 & .203 & .001 & .911 & .215 & .001 \\
\hline \multicolumn{10}{|l|}{ Haupteffekte } \\
\hline AE Dummy & & & & .367 & .472 & .441 & .306 & .457 & .508 \\
\hline KG Dummy & & & & .573 & .487 & .247 & .557 & .469 & .243 \\
\hline Autonomiemotiv (AutMot) & & & & .362 & .200 & .077 & 1.097 & .373 & .006 \\
\hline \multicolumn{10}{|l|}{ Interaktionen } \\
\hline AE Dummy $\times$ AutMot & & & & & & & 1.076 & .504 & .039 \\
\hline KG Dummy $\times$ AutMot & & & & & & & .954 & .502 & .065 \\
\hline$\Delta R^{2}$ & & .166 & & & .103 & & & .089 & \\
\hline$F$ for change in $R^{2}$ & & 8.568 & & & 1.882 & & & 2.638 & \\
\hline
\end{tabular}

Anmerkung: Die Gruppen wurden mithilfe von zwei Dummy Variablen mit 0 (Autonomieunterstützung) als Referenzgruppe codiert.

diererinnen ausgewertet, die eine Übereinstimmung (Intraklassenkoeffizient, ICC) von .91 erzielen. Die Autonomiewerte lagen zwischen 0 und $12(M=5.44, S D=3.95)$. Nach einer Empfehlung von Schultheiss und Pang (2007) wurden die mit der Wortanzahl korrelierenden Rohwerte bereinigt (residualisierte Autonomie-Rohwerte).

Intrinsische Motivation. Intrinsische Motivation wurde mithilfe der Skala Interesse/Freude aus dem Intrinsic Motivation Inventory (Ryan, 1982) erhoben. Die Teilnehmenden sollten hierbei Aussagen wie "Während ich an der Aufgabe arbeitete, dachte ich darüber nach, wie sehr mir das Spaß macht" auf einer siebenstufigen Skala (1 = trifft überhaupt nicht zu, 7 = trifft voll und ganz zu) beurteilen. Das Cronbachs Alpha war in der vorliegenden Studie zufriedenstellend hoch (T1: .81, T2: .86).

\section{Ergebnisse}

\section{Vorabanalysen, Korrelationen und deskriptive Statistik}

Mädchen und Jungen unterschieden sich in keiner der erfassten Variablen. Auch das Alter der Probanden hatte keinen Einfluss auf die erfassten Variablen. Weiter finden sich keine Unterschiede zwischen den Gruppen in der Ausprägung des impliziten Autonomiemotivs, $F(2,42)=$ 1.13, $p=.33$. Das implizite Autonomiemotiv (Rohwert: $M=5.44, S D=3.95$; wortanzahlkorrigierter Wert: $M=.02$, $S D=.99)$ und die intrinsische Motivation zu T1 $(M=4.28$, $S D=1.43)$, und zu T2 $(M=4.31, S D=1.42)$ korrelierten nicht (T1: $r=-.19, p=.21$; T2: $r=.20, p=.20$ ). Signifikante Korrelationen fanden sich für die zweimalige Messung von intrinsischer Motivation, $r=.41, p=.005$. Die erreichte Punktzahl während der Übung hatte zudem keinen Einfluss auf die intrinsische Motivation, $r=.15, p=.32$.

\section{Moderationsanalyse}

Zur Prüfung der Hypothese wurde eine hierarchische Regressionsanalyse mit intrinsischer Motivation zum Zeitpunkt T2 als Kriterium durchgeführt. Im ersten Modell wurde für die Baseline-Messung (T1) der intrinsischen Motivation kontrolliert. Das zweite Modell beinhaltet die experimentellen Gruppen sowie den Moderator (implizites Autonomiemotiv) zur Prüfung der Haupteffekte. Keine dieser Variablen war im Modell 2 signifikanter Prädiktor intrinsischer Motivation (siehe Tabelle 1). Im dritten Modell wurden die Interaktionsterme der einzelnen Gruppen mit dem impliziten Autonomiemotiv hinzugefügt. Um die Interaktion zwischen den drei experimentellen Gruppen (AF, AE, KG) und dem Autonomiemotiv zu testen, wurden die beiden Dummy-Variablen AE-Dummy (O für AF, 1 für AE, 0 für KG) und KG-Dummy (0 für AF, 0 für AE, 1 für KG) erstellt. Die Interaktion AE-Dummy $\times$ Autonomiemotiv wurde signifikant, $b=$ $-1.08, s e_{\mathrm{b}}=.50 p=.039$. Die Interaktion KG-Dummy $\times$ Autonomiemotiv hingegen wurde nur marginal signifikant, $b=-.95, s e_{b}=.50, p=.065$. Tabelle 1 zeigt die Details der Regressionsanalyse auf.

Die Darstellung in Abbildung 1 zeigt die Richtung der Interaktion auf. Sie zeigt auch die Ergebnisse ergänzender Analysen zur Prüfung statistischer Unterschiede zwischen den Balken (Johnson-Neyman Technik, siehe Aiken \& West, 1991, S. 132). 


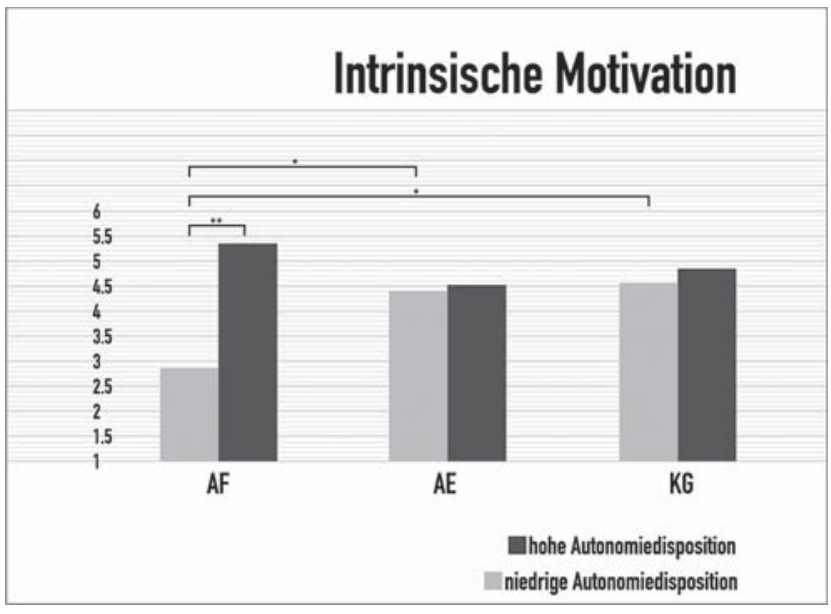

Anmerkungen: ${ }^{*} p<.05,{ }^{* *} p<.01$.

Abbildung 1. Effekt der experimentellen Manipulation (AF Autono mieförderung, AE Autonomieeinschränkung, KG Kontrollgruppe) in Abhängigkeit von der impliziten Autonomiedisposition der Schülerin nen und Schüler auf Intrinsische Motivation.

Personen mit starkem Autonomiemotiv (eine Standardabweichung über dem Mittelwert) berichten in der Autonomiebedingung signifikant mehr intrinsische Motivation als Personen mit schwachem Autonomiemotiv (eine Standardabweichung unter dem Mittelwert), $b=1.29$, $s e_{b}=.36, p=.003$. Jedoch unterscheiden sich Personen mit einem hohen Autonomiemotiv weder im Vergleich zwischen Autonomieförderung und Kontrollgruppe, $b=$ $-.40, s e_{b}=.68, p=.563$, noch im Vergleich zwischen Autonomieförderung und Autonomieeinschränkung, $b=-.77$, $s e_{b}=.69, p=.268$, in ihrer intrinsischen Motivation.

Personen mit einem niedrigen Autonomiemotiv gaben hingegen mehr intrinsische Motivation in der Bedingung Autonomieeinschränkung im Vergleich zur Autonomieförderung, $b=1.38, s e_{b}=.66, p=.048$, als auch im Vergleich der Kontrollbedingung im Vergleich zur Autonomieförderung an, $b=1.51, s e_{b}=.70, p=.036$.

\section{Diskussion}

Die Ergebnisse der Studie zeigen, dass Autonomieförderung durch das soziale Umfeld nicht für alle Personen gleichermaßen gut $\mathrm{zu}$ intrinsischer Motivation führt. Vergleicht man Personen mit hohem und niedrigem Autonomiemotiv in der Bedingung Autonomieförderung, zeigt sich, dass Schülerinnen und Schüler mit einem hohen impliziten Autonomiemotiv signifikant mehr intrinsische Motivation aufzeigen als Schülerinnen und Schüler mit einem niedrigen impliziten Auto- nomiemotiv (siehe Abbildung 1). Dies unterstützt das Verständnis von impliziten Motiven als die Fähigkeit korrespondierende Anreize als belohnend wahrzunehmen (Schultheiss \& Hale, 2007).

In der autonomieeinschränkenden Bedingung traten entgegen unserer Annahme keine Unterschiede zwischen hoch und niedrig Autonomiemotivierten auf. Dies könnte darauf zurückzuführen sein, dass die Manipulation in der autonomieeinschränkenden Bedingung nicht autonomie-restriktiv genug war. Für diese Annahme spricht auch, dass das Interaktionsmuster keinen Unterschied zwischen der autonomieeinschränkenden Gruppe und der Kontrollgruppe aufzeigte. Etwas spekulativ formuliert, könnte angenommen werden, dass ein gewisser Grad an Kontrolle und als Konsequenz auch an Autonomieeinschränkung im Sportunterricht die Norm darstellt und deshalb keine Unterschiede zwischen der Autonomieeinschränkung und der Kontrollgruppe gefunden werden konnten. Zusammenfassend lässt sich der differentielle Effekt von Autonomieunterstützung festhalten: Nur Personen mit einem hohen Autonomiemotiv, nicht aber Personen mit schwachem Autonomiemotiv profitierten von Autonomieunterstützung im Schulsport. Dies entspricht vorliegenden Befunden zur Passungshypothese (Schüler \& Brandstätter, 2013; Schüler et al., 2010, 2013, 2014; Sheldon \& Schüler, 2011) und erweitert die Selbstbestimmungstheorie (Deci \& Ryan, 1985) um den Punkt, dass Personenmerkmale wie individuelle Motivunterschiede relevant für die Erklärung von positiven Effekten autonomiefördernder Sportumwelten sind. Mit dieser Studie wurde nun erstmalig der differentielle Effekt von Autonomie auch für den Schulsport aufgezeigt.

Unsere Befunde haben vor allem eine relevante praktische Implikation. Sie zeigen, dass eine Gruppe von Schülern und Schülerinnen solche mit schwachem Autonomiemotiv nicht von Autonomieförderung durch die Lehrperson profitieren. Beachtet man die Resultate für Personen mit einem niedrigen Autonomiemotiv, zeigt sich sogar, dass Autonomie negative Effekte auf intrinsische Motivation haben kann. Eine mögliche Erklärung hierfür könnte sein, dass Schülerinnen und Schüler mit einem niedrigen Autonomiemotiv zu viel Autonomie als negativ erleben. Dieser Befund kann mit der Hidden Stressor Hypothese (Baumann, Kaschel \& Kuhl, 2005) erklärt werden. Diese besagt, dass Personen einen intrapsychischen Konflikt erleben, wenn ihr implizites Motiv nicht mit einem expliziten Ziel übereinstimmt, was zu erhöhtem Stress und vermindertem Wohlbefinden führen kann. Ein ähnliches Befundmuster wurde für das implizite Autonomiemotiv bereits auf physiologischen Stress (AlphaAmylase im Speichel) in einer Studie mit Vignetten von Sieber und Kollegen gefunden (Sieber et al., 2016). So 
zeigte sich in der Studie, dass Jugendliche mit einem niedrigen Autonomiemotiv, die sich in Szenarien versetzen mussten, die eine autonomiefördernde Sportlehrperson beinhalteten, eine erhöhte physiologische Stressreaktion aufzeigten. Eine weitere Erklärungsmöglichkeit könnte darin liegen, dass die Schülerinnen und Schüler in einer Situation die nur die Befriedigung eines Motivs bedient, keine Möglichkeit besitzen, die anderen Motive (z. B. Anschlussmotiv) zu befriedigen. Für diese Personengruppe müssen demnach andere Anreize in der Sportsituation gefunden werden, die intrinsische Motivation zum Sporttreiben erhöhen können. Es liegt nahe, ein differenziertes Motivprofil, bestehend aus dem Leistungs-, Anschluss-, Macht- und Autonomiemotiv zu erstellen und sportsituative Anreize maßgeschneidert zu schaffen.

Eine Möglichkeit hierzu sind maßgeschneiderte Coachingmaßnahmen und Beratungen, ganz im Sinne einer „tailored-intervention" wie sie in der Gesundheitspsychologie und Medizin breit diskutiert wird (Noar, Benac \& Harris, 2007; Ryan \& Lauver, 2002). Falls direktes individuelles Coaching nicht möglich ist wie beispielsweise im Schulsetting ist das Kreieren von Sportumwelten, die die Befriedigung aller Basisbedürfnisse (also Autonomie, Kompetenz und Zugehörigkeit) zulassen, zu empfehlen. Dies mit dem Hintergrundwissen, dass Personen sich hinsichtlich ihrer impliziten Motive (Autonomie, Anschluss und Leistung) unterscheiden und unterschiedlich auf die Befriedigung der Basisbedürfnisse (Autonomie, Zugehörigkeit und Kompetenz) reagieren (Schüler et al., 2010, 2013, 2014; Sheldon \& Schüler, 2011, Sieber et al., 2016). Ein Angebot mit breitem Anreizspektrum ermöglicht also die Befriedigung verschiedener Motive.

Die vorliegende Studie reiht sich ein in verschiedene Forschungsergebnisse, die zeigen, dass intrinsische Motivation, Wohlbefinden und sportliche Leistung insbesondere dann vorteilhaft ausfallen, wenn Anreize der sportlichen Umwelt zu den Motiven einer Person passen.

\section{Limitationen}

Um die experimentelle Manipulation in den Schulunterricht einzubauen, wurde ein Setting kreiert, in dem die Schülerinnen und Schüler einzeln aus dem Basketballspiel gerufen wurden. Dies hatte den Vorteil, dass die Interaktion mit den Schülerinnen und Schülern nicht durch konfundierende Variablen während des Unterrichts beeinflusst wurde. Jedoch kann nicht ausgeschlossen werden, dass der Verlauf des vorangehenden Basketballspiels die Jugendlichen beeinflusst hat. Die Intervention wurde aus organisatorischen Gründen auf drei Minuten begrenzt und bezog sich auf eine spezifische Übung. In künftigen Studien wäre zum Beispiel ein Szenario denkbar, in dem sich Sportlehrpersonen über eine ganze Lektion hinweg entweder autonomiefördernd oder autonomieeinschränkend verhalten.

Weiter würde künftige Forschung von einer Replikation mit einer grösseren Stichprobe profitieren. Die vorliegende Studie ist die erste die aufzeigt, dass die Passungshypothese von Schüler und Kollegen (2014) auch im Schulsport einen Einfluss auf Motivation besitzt, weist jedoch eine geringe Stichprobengrösse auf. Deshalb sind die Resultate insbesondere hinsichtlich Empfehlungen für die Praxis als erste Tendenzen zu verstehen. Eine Replikation mit einer grossen Stichprobe würde sich weiter anbieten, um negative Effekte von Autonomie auf Personen mit einem niedrigen Autonomiemotiv und die dahinterliegenden Mechanismen (z. B. Hidden Stressor Hypothese) zu untersuchen.

\section{Literatur}

Aiken, L. S., West, S. G. \& Reno, R. R. (1991). Multiple regression: Testing and interpreting interactions. Newbury Park, CA: Sage. Adie, J., Duda, J. L. \& Ntoumanis, N. (2008). Autonomy support, basic need satisfaction and the optimal functioning of adult male and female sport participants: A test of basic needs theory. Motivation and Emotion, 32, 189 - 199.

Adie, J., Duda, J. L. \& Ntoumanis, N. (2012). Perceived coach autonomy support, basic need satisfaction and the well- and illbeing of elite youth soccer players: A longitudinal investigation. Psychology of Sport and Exercise, 13, 1-32.

Baard, P. P., Deci, E. L. \& Ryan, R. M. (2004). Intrinsic need satisfaction: A motivational basis of performance and well-being in two work settings. Journal of Applied Social Psychology, 34, $2045-2068$

Baumann, N., Kaschel, R. \& Kuhl, J. (2005). Striving for unwanted goals: Stress dependent discrepancies between explicit and implicit achievement motives reduce subjective well-being and increase psychosomatic symptoms. Journal of Personality and Social Psychology, 89, 781-799. doi: 10.1037/00223514.89.5.781

Caspersen, C. J., Pereira, M. A. \& Curran, K. M. (2000). Changes in physical activity patterns in the United States, by sex and cross-sectional age. Medicine and Science in Sports and Exercise, 32, $1601-1609$.

DeCharms, R. (1968). Personal causation. New York, NY: Academic Press.

DeCharms, R. \& Plimpton, F. (1992). The origin scoring system. In C. R. Smith (Ed.), Motivation and personality: Handbook of thematic content analysis (pp. 334-375). Cambridge, UK: Cambridge University Press.

Deci, E. L. \& Ryan, R. M. (1985). Intrinsic motivation and self-determination in human behaviour. New York, NY: Plenum.

Deci, E. L. \& Ryan, R. M. (2000). The "what" and "why" of goal pursuits: Human needs and the self-determination of behavior. Psychological Inquiry, 11, $227-268$. 
Edmunds, J., Ntoumanis, N. \& Duda, J. L. (2006). A test of selfdetermination theory in the exercise domain. Journal of Applied Social Psychology, 36, 2240-2265.

Fenton, S. A. M., Duda, J. L. \& Barrett, T. (2016). Optimising physical activity engagement during youth sport: a self-determination theory approach. Journal of Sports Sciences, 34, 1874-1884.

Fuchs, R. (2003). Sport, Gesundheit und public health (S. 273). Göttingen: Hogrefe.

Güntert, S. T. (2015). The impact of work design, autonomy support, and strategy on employee outcomes: A differentiated perspective on self-determination at work. Motivation and Emotion, 39, 74-87.

Hagger, M. S. \& Chatzisarantis, N. L. (2015). The trans-contextual model of autonomous motivation in education: Conceptual and empirical issues and meta-analysis. Review of Educational Research, 86, 360-407.

Hofer, J. \& Busch, H. (2011). Satisfying one's needs for competence and relatedness: Consequent domain-specific well-being depends on strength of implicit motives. Personality and Social Psychology Bulletin, 37, $1147-1158$.

How, Y., Whipp, P., Dimmock, J. \& Jackson, B. (2013). The effects of choice on autonomous motivation, perceived autonomy support, and physical activity levels in high school physical education. Journal of Teaching in Physical Education, 32, 131-148.

Lamprecht, M., Fischer, A. \& Stamm, H. P. (2014). Sport Schweiz 2014: Sportaktivität und Sportinteresse der Schweizer Bevölkerung (Bericht). Magglingen: Bundesamt für Sport BASPO.

Lewin, K. (1931). Die psychologische Situation bei Lohn und Strafe. Leipzig: Hirsel.

Lewin, K. (1936). Principles of topological psychology. New York, NY: McGraw-Hill.

Lynch, M. F., Plant, R. W. \& Ryan, R. M. (2005). Psychological needs and threat to safety: Implications for staff and patients in a psychiatric hospital for youth. Professional Psychology: Research and Practice, 36, 415-425.

McClelland, D. C. (1985). Human motivation. Glenview, IL: Foresman \& Co.

Nie, Y., Chua, B. L., Yeung, A. S., Ryan, R. M. \& Chan, W. Y. (2015). The importance of autonomy support and the mediating role of work motivation for well-being: Testing self-determination theory in a chinese work organization. International Journal of Psychology, 50, 245-255.

Noar, S. M., Benac, C. N. \& Harris, M. S. (2007). Does tailoring matter? Meta-analytic review of tailored print health behavior change interventions. Psychological Bulletin, 133, 673-693.

Ryan, P. \& Lauver, D. R. (2002). The efficacy of tailored interventions. Journal of Nursing Scholarship, 34, $331-337$.

Ryan, R. M. (1982). Control and information in the intrapersonal sphere: An extension of cognitive evaluation theory. Journal of Personality and Social Psychology, 43, 450-461.

Ryan, R. M. \& Deci, E. L. (2008). Self-determination theory and the role of basic psychological needs in personality and the organization of behavior. In O. P. John, R. W. Robins \& L. A. Pervin (Eds.), Handbook of personality. Theory and research (pp. 654678). New York, NY: Guilford Press.

Schüler, J. \& Brandstätter, V. (2013). How basic need satisfaction and dispositional motives interact in predicting flow experience in sport. Journal of Applied Social Psychology, 43, 687-705.

Schüler, J., Brandstätter, V. \& Sheldon, K. M. (2013). Do implicit motives and basic psychological needs interact to predict wellbeing and flow? Testing a universal hypothesis and a matching hypothesis. Motivation and Emotion, 37, 480 - 495.

Schüler, J., Sheldon, K. M. \& Fröhlich, S. M. (2010). Implicit need for achievement moderates the relationship between competence need satisfaction and subsequent motivation. Journal of Research in Personality, 44, 1-12.
Schüler, J., Sheldon, K. M., Prentice, M. \& Halusic, M. (2014). Do some people need autonomy more than others? Implicit dispositions toward autonomy moderate the effects of felt autonomy on well-being. Journal of Personality, 84, 5-20.

Schultheiss, O. C. \& Hale, J. A. (2007). Implicit motives modulate attentional orienting to facial expressions of emotion. Motivation and Emotion, 31, $13-24$.

Schultheiss, O. C. \& Pang, J. S. (2007). Measuring implicit motives. In R. W. Robins, R. C. Fraley \& R. Krueger (Eds.), Handbook of research methods in personality psychology (pp. $322-344)$. New York, NY: Guilford.

Sheldon, K. M. \& Krieger, L. S. (2007). Understanding the negative effects of legal education on law students: A longitudinal test of self-determination theory. Personality and Social Psychology Bulletin, 33, $883-897$.

Sheldon, K. M. \& Schüler, J. (2011). Wanting, having, and needing: Integrating motive disposition theory and self-determination theory. Journal of Personality and Social Psychology, 101, $1106-1123$.

Sieber, V., Schüler, J. \& Wegner, M. (2016). The effects of autonomy on salivary alpha-amylase: The role of individual differences. Psychoneuroendocrinology, 74, 173-178. doi: 10.1016/ j.psyneuen.2016.09.003.

Telama, R. \& Yang, X. (2000). Decline of physical activity from youth to young adulthood in Finland. Medicine and Science in Sports and Exercise, 32, $1617-1622$.

Van Mechelen, W., Twisk, J. W. R., Post, G. B., Snel, J. \& Kemper, H. C. G. (2000). Physical activity of young people: The Amsterdam Longitudinal Growth and Health Study. Medicine and Science in Sports and Exercise, 32, $1610-1616$.

Vansteenkiste, M., Simons, J., Lens, W., Soenens, B. \& Matos, L. (2005). Examining the motivational impact of intrinsic versus extrinsic goal framing and autonomy-supportive versus internally controlling communication style on early adolescents' academic achievement. Child Development, 76, 483-501.

Williams, G. C., Grow, V., Freedman, Z. R., Ryan, R. M. \& Deci, E. L. (1996). Motivational predictors of weight loss and weight-loss maintenance. Journal of Personality and Social Psychology, 70, $115-126$.

Williams, G. C., Niemiec, C. P., Patrick, H., Ryan, R. M. \& Deci, E. L. (2009). The importance of supporting autonomy and perceived competence in facilitating long- term tobacco abstinence. Annals of Behavioral Medicine, 37, 315-324.

\section{Vanda Sieber}

Universität Zürich

Psychologisches Institut

Binzmühlestrasse 14/11

8050 Zürich

Schweiz

sieber@psychologie.uzh.ch

\section{PD Dr. Mirko Wegner}

Universität Bern

Institut für Sportwissenschaft

Bremgartenstraße 145

3012 Bern

Schweiz

\section{Prof. Dr. Julia Schüler}

Universität Konstanz

Institut für Sportwissenschaft

Universitätsstraße 10

78464 Konstanz

Deutschland 